\title{
Two scenarios for superconductivity in $\mathrm{CeRh}_{2} \mathrm{As}_{2}$
}

\author{
David Möckli ${ }^{1}$ and Aline Ramires ${ }^{2}$ \\ ${ }^{1}$ Instituto de Física, Universidade Federal do Rio Grande do Sul, 91501-970 Porto Alegre, Brazil \\ ${ }^{2}$ Paul Scherrer Institut, CH-5232 Villigen PSI, Switzerland
}

(Received 23 February 2021; accepted 18 May 2021; published 11 June 2021)

\begin{abstract}
$\mathrm{CeRh}_{2} \mathrm{As}_{2}$, a nonsymmorphic heavy fermion material, was recently reported to host a remarkable temperature versus $z$-axis magnetic-field phase diagram with two superconducting phases. In this material, the two inequivalent $\mathrm{Ce}$ sites per unit cell, related by inversion symmetry, introduce a sublattice structure corresponding to an extra internal degree of freedom. Here we propose a classification of the possible superconducting states in $\mathrm{CeRh}_{2} \mathrm{As}_{2}$ from the two Ce-sites' perspective. Based on the superconducting fitness analysis and the quasiclassical Eilenberger equations, we discuss two limits: Rashba spin-orbit coupling and interlayer hopping dominated normal state. In both limits, we are able to find two scenarios that generate phase diagrams in qualitative agreement with experiments: (i) intrasublattice pairing with an even-odd transition under $z$-axis magnetic field and (ii) intersublattice pairing with an odd-odd transition under $z$-axis magnetic field.
\end{abstract}

DOI: 10.1103/PhysRevResearch.3.023204

\section{INTRODUCTION}

The heavy fermion $\mathrm{CeRh}_{2} \mathrm{As}_{2}$ was recently discovered to host remarkable properties in the superconducting state [1]. Under $z$-axis magnetic field, a transition between a lowfield and a high-field superconducting phase is observed by measurements of magnetization and magnetostriction. The presence of two superconducting phases is unusual, and has only been reported in other two stoichiometric heavy fermion materials: $\mathrm{UPt}_{3}$ [2-4] and $\mathrm{UTe}_{2}$ [5]. The high effective electronic mass inferred from the low-temperature value of the specific heat coefficient indicates that $\mathrm{CeRh}_{2} \mathrm{As}_{2}$ is in the heavy fermion regime, but the power-law temperature dependence of the specific heat below $4 \mathrm{~K}$ suggests the proximity to a quantum critical point, whose fluctuations can play an important role for pairing [6-9]. Furthermore, the high-field phase has an upper critical field of $14 \mathrm{~T}$, exceptional for a material with a superconducting critical temperature $\left(T_{c}\right)$ of $0.26 \mathrm{~K}$, suggesting that $\mathrm{CeRh}_{2} \mathrm{As}_{2}$ hosts an unconventional triplet superconducting state.

Theory developed in the context of layered superconductors with local inversion symmetry breaking accounts for the qualitative features of the temperature versus magnetic field phase diagram of $\mathrm{CeRh}_{2} \mathrm{As}_{2}$ [10-12]. More recently, these studies were supplemented by detailed investigations including also orbital depairing effects $[13,14]$. Intralayer singlet pairing (referred to here as BCS) is assumed to be the stable superconducting state within each layer. Once a magnetic field is applied perpendicular to the layers, a pair-density wave

Published by the American Physical Society under the terms of the Creative Commons Attribution 4.0 International license. Further distribution of this work must maintain attribution to the author(s) and the published article's title, journal citation, and DOI.
(PDW) state, with a sign change of the order parameter between layers, is favored under the requirement that Rashba spin orbit coupling (SOC) is comparable to interlayer hopping (ILH) amplitudes.

Nevertheless, the phenomenology of $\mathrm{CeRh}_{2} \mathrm{As}_{2}$ indicates that other scenarios might be possible. In particular, the small anisotropy of the effective mass, inferred from the slope of the upper critical field around $T_{c}$, indicates that the system is rather three-dimensional [1]. This is in agreement with recent first-principles calculations [15], and with other 122 materials in this family. As an example, $\mathrm{CeCu}_{2} \mathrm{Si}_{2}$ crystallizes in the $\mathrm{ThCr}_{2} \mathrm{Si}_{2}$-type structure (the centrosymmetric analog of the $\mathrm{CaBa}_{2} \mathrm{Ge}_{2}$-type structure of $\mathrm{CeRh}_{2} \mathrm{As}_{2}$ ), displays a three-dimensional spin density wave state supported by Fermi surface nesting, and hosts superconductivity around the pressure-induced quantum critical point $[16,17]$. Motivated by these facts, here we analyze the possible unconventional superconducting states that can be hosted by $\mathrm{CeRh}_{2} \mathrm{As}_{2}$. We start with focus on the main ingredient, the Ce ions, and propose a microscopic model for the normal-state Hamiltonian based on the spin and sublattice (SL) degrees of freedom (DOF). This model allows us to discuss two limits: (i) two-dimensional, dominated by Rashba-SOC and (ii) three-dimensional, dominated by ILH. We then classify all possible types of Cooper pairs that can be formed in this material. We perform the superconducting fitness analysis and discuss the relative stability of different superconducting states in both limits. Finally, we obtain temperature versus magnetic-field phase diagrams from the quasiclassical Eilenberger equations, based on which we can propose two scenarios for the temperature versus $z$ axis magnetic field phase diagram of $\mathrm{CeRh}_{2} \mathrm{As}_{2}$.

\section{THE MODEL}

$\mathrm{CeRh}_{2} \mathrm{As}_{2}$ has a $\mathrm{CaBa}_{2} \mathrm{Ge}_{2}$-type structure with the nonsymmorphic centrosymmetric space group P4/nmm (No. 


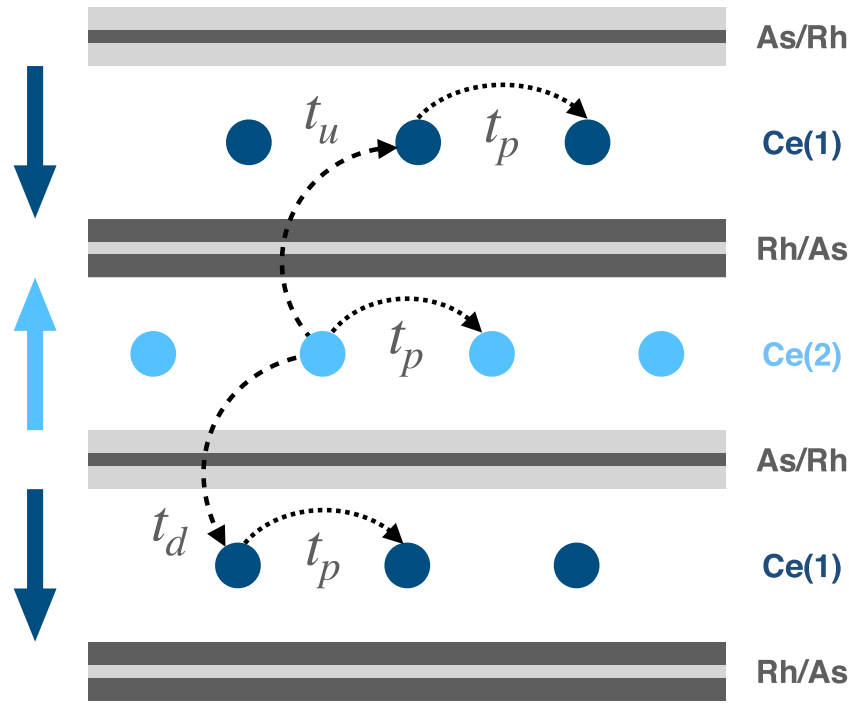

FIG. 1. Schematic depiction of $\mathrm{CeRh}_{2} \mathrm{As}_{2}$. The spheres correspond to the two inequivalent $\mathrm{Ce}$ atoms and the rectangular structures to the two types of Rh/As layers. The big arrows on the left correspond to the effective electrical field generating a staggered Rashba-SOC. The dotted lines correspond to in-plane intra-SL hopping with amplitude $t_{p}$ (same for all layers), and the dashed lines correspond to inter-SL hopping, with amplitude $t_{u}$ or $t_{d}$, depending on if the hopping is to a neighbor in the layer above or below.

129). Given the heavy fermion nature of the electronic structure around the Fermi energy inferred by recent experiments, we start modeling the electronic DOF from the Ce sites' perspective. The Ce atoms are located in between Rh-As layers, which appear intercalated in two flavors: with Rh atoms tetrahedrally coordinated by As, or vice versa, as schematically shown in Fig. 1. The intercalation of two types of Rh-As layers generates two inequivalent $\mathrm{Ce}$ sites with $C_{4 v}$ point group symmetry. Importantly, the $\mathrm{Ce}$ sites are not centers of inversion. The point group $C_{4 v}$ can be generated by $C_{4 z}$, a rotation along the $z$-axis by $\pi / 2$, and $\sigma_{x z}$, a mirror reflection along the $x z$-plane. The complete space group includes inversion, which can be made a symmetry at the Ce sites if composed with half-integer translation vectors. We define $i_{1 / 2}$ as inversion composed with a translation by $(a / 2, a / 2, c / 2)$, which links the two inequivalent $\mathrm{Ce}$ sites (here $a$ and $c$ are the unit cell dimensions in the plane and along the $z$-axis, respectively). These three operations generate the complete space group. Here we note that this space group is isomorphic to $D_{4 h}$ up to integer lattice translations [18]. Given these generators, Table I summarizes the properties of the irreducible representations. For simplicity, here we focus on the symmetry analysis around the $\Gamma$ point.

We start with the most general noninteracting normal-state Hamiltonian considering Wannier functions localized at the Ce atoms accounting for a two SL structure

$$
\mathcal{H}_{0}=\sum_{\mathbf{k}} \hat{\Psi}_{\mathbf{k}}^{\dagger} \hat{H}_{0}(\mathbf{k}) \hat{\Psi}_{\mathbf{k}},
$$

where $\hat{\Psi}^{\dagger}=\left(c_{1 \uparrow}^{\dagger}, c_{1 \downarrow}^{\dagger}, c_{2 \uparrow}^{\dagger}, c_{2 \downarrow}^{\dagger}\right)$ encodes the two $\operatorname{SLs}(1,2)$ and the $\operatorname{spin}(\uparrow, \downarrow)$ DOF. The $4 \times 4$ matrix $\hat{H}_{0}(\mathbf{k})$ can be
TABLE I. Irreducible representations (irrep) at the $\Gamma$-point associated with the three symmetry operations at the Ce sites. The last column shows examples of polynomials in each irrep.

\begin{tabular}{lcccc}
\hline \hline Irrep & $C_{4 z}$ & $\sigma_{x z}$ & $i_{1 / 2}$ & Basis \\
\hline$A_{1 g}$ & +1 & +1 & +1 & $x^{2}+y^{2}, z^{2}$ \\
$A_{2 g}$ & +1 & -1 & +1 & $x y\left(x^{2}-y^{2}\right)$ \\
$B_{1 g}$ & -1 & +1 & +1 & $x^{2}-y^{2}$ \\
$B_{2 g}$ & -1 & -1 & +1 & $x y$ \\
$E_{g}$ & 0 & 0 & +2 & $\{x z, y z\}$ \\
$A_{1 u}$ & +1 & +1 & -1 & $z$ \\
$A_{2 u}$ & +1 & -1 & -1 & $x y z\left(x^{2}-y^{2}\right)$ \\
$B_{1 u}$ & -1 & +1 & -1 & $z\left(x^{2}-y^{2}\right)$ \\
$B_{2 u}$ & -1 & -1 & -1 & $x y z$ \\
$E_{u}$ & 0 & 0 & -2 & $\{x, y\}$ \\
\hline \hline
\end{tabular}

parametrized as

$$
\hat{H}_{0}(\mathbf{k})=\sum_{a, b} h_{a b}(\mathbf{k}) \hat{\tau}_{a} \otimes \hat{\sigma}_{b},
$$

where $\hat{\tau}_{a}$ and $\hat{\sigma}_{b}$ are Pauli matrices, $\{a, b\}=\{1,2,3\}$, or the two-dimensional identity matrix, $\{a, b\}=\{0\}$, corresponding to the SL and spin DOF, respectively. In the presence of inversion (implemented as $\tau_{1} \otimes \sigma_{0}$ accompanied by $\mathbf{k} \rightarrow-\mathbf{k}$ ) and time-reversal symmetry (implemented as $i \tau_{0} \otimes \sigma_{2}$, accompanied by complex conjugation and $\mathbf{k} \rightarrow-\mathbf{k})$, only the $(a, b)$ pairs summarized in Table II are symmetry allowed.

We now associate each term with specific physical processes. $(0,0)$ concerns intra-SL hopping, with $h_{00}(\mathbf{k})=2 t_{p}\left[\cos \left(k_{x} a\right)+\cos \left(k_{y} a\right)\right]$, dominated by intralayer hopping to nearest neighbors with amplitude $t_{p}$. $(1,0)$ and $(2,0)$ stem from inter-SL hopping, which in this case is necessarily out-of-plane, with $h_{10}(\mathbf{k})=$ $4\left(t_{u}+t_{d}\right) \cos \left(k_{x} a / 2\right) \cos \left(k_{y} a / 2\right) \cos \left(k_{z} c / 2\right)$ and $h_{20}(\mathbf{k})=$ $-4\left(t_{u}-t_{d}\right) \cos \left(k_{x} a / 2\right) \cos \left(k_{y} a / 2\right) \sin \left(k_{z} c / 2\right)$. These terms are parametrized by $t_{u}$ and $t_{d}$, the hopping amplitudes to the nearest neighbors in the layer above and below, which are inevitably distinct due to inversion symmetry breaking. $(3,1)$ and $(3,2)$ are concerned with intra-SL staggered Rashba-type SOC, $\quad h_{31}(\mathbf{k})=-\alpha \sin \left(k_{y} a\right) \quad$ and $\quad h_{32}(\mathbf{k})=\alpha \sin \left(k_{x} a\right)$, parametrized by $\alpha$. Finally, $(3,3)$ is an Ising-type SOC, $h_{33}(\mathbf{k})=\lambda \sin \left(k_{x} a\right) \sin \left(k_{y} a\right) \sin \left(k_{z} c\right)\left[\cos \left(k_{x} a\right)-\cos \left(k_{y} a\right)\right]$, associated with hopping to neighbors of the same SL in the next nearest layer, parametrized by $\lambda$. This Hamiltonian is the same as the one proposed in Ref. [1].

TABLE II. Symmetry-allowed $(a, b)$ terms in the normal state Hamiltonian, as given in Eq. (2). The table highlights the irrep, the even or odd $\mathbf{k}$ dependence, the associated physical process, and dominant parameter for each term.

\begin{tabular}{lcccc}
\hline \hline$(a, b)$ & Irrep & $\mathbf{k}$ & Process & Parameter \\
\hline$(0,0)$ & $A_{1 g}$ & Even & Intra-SL hopping & $t_{p}$ \\
$(1,0)$ & $A_{1 g}$ & Even & Inter-SL hopping & $t_{u}+t_{d}$ \\
$(2,0)$ & $A_{1 u}$ & Odd & Inter-SL hopping & $t_{u}-t_{d}$ \\
$\{(3,1),(3,2)\}$ & $E_{u}$ & Odd & Rashba-SOC & $\alpha$ \\
$(3,3)$ & $A_{2 u}$ & Odd & Ising-SOC & $\lambda$ \\
\hline \hline
\end{tabular}


TABLE III. Symmetry classification of the $[a, b]$ matrices associated with all order parameters, defined in Eq. (3), organized by irreducible representations (irreps) around the $\Gamma$ point. Here $E / O$ stands for even/odd and S/T for singlet/triplet. The irrep associated with the complete order parameter is obtained by taking the product with the irrep of $d_{a b}(\mathbf{k})$, which is always nontrivial for the odd-k order parameters.

\begin{tabular}{cccccc}
\hline \hline Irrep & {$[a, b]$} & Spin & SL & $\mathbf{k}$ & Parity \\
\hline$A_{1 g}$ & {$[0,0]$} & $\mathrm{S}$ & Intra & $\mathrm{E}$ & $\mathrm{E}$ \\
& {$[1,0]$} & $\mathrm{S}$ & Inter & $\mathrm{E}$ & $\mathrm{E}$ \\
$A_{2 g}$ & {$[0,3]$} & $\mathrm{T}$ & Intra & $\mathrm{O}$ & $\mathrm{O}$ \\
& {$[1,3]$} & $\mathrm{T}$ & Inter & $\mathrm{O}$ & $\mathrm{O}$ \\
$A_{1 u}$ & {$[3,0]$} & $\mathrm{S}$ & Intra & $\mathrm{E}$ & $\mathrm{O}$ \\
& {$[2,0]$} & $\mathrm{S}$ & Inter & $\mathrm{O}$ & $\mathrm{E}$ \\
$A_{2 u}$ & {$[2,3]$} & $\mathrm{T}$ & Inter & $\mathrm{E}$ & $\mathrm{O}$ \\
& {$[3,3]$} & $\mathrm{T}$ & Intra & $\mathrm{O}$ & $\mathrm{E}$ \\
$E_{g}$ & $\{[0,1],[0,2]\}$ & $\mathrm{T}$ & Intra & $\mathrm{O}$ & $\mathrm{O}$ \\
& $\{[1,1],[1,2]\}$ & $\mathrm{T}$ & Inter & $\mathrm{O}$ & $\mathrm{O}$ \\
$E_{u}$ & $\{[2,1],[2,2]\}$ & $\mathrm{T}$ & Inter & $\mathrm{E}$ & $\mathrm{O}$ \\
& $\{[3,1],[3,2]\}$ & $\mathrm{T}$ & Intra & $\mathrm{O}$ & $\mathrm{E}$ \\
\hline \hline
\end{tabular}

The superconducting order parameter can be written in a similar fashion:

$$
\hat{\Delta}(\mathbf{k})=\sum_{a, b} d_{a b}(\mathbf{k}) \hat{\tau}_{a} \otimes \hat{\sigma}_{b}\left(i \sigma_{2}\right) .
$$

In the presence of inversion symmetry, we can distinguish between even or odd in $\mathbf{k}$ and even or odd parity gap functions. Table III lists all the order parameters, their nature in terms of the microscopic DOFs, and their symmetry properties. The different brackets distinguish the normal-state Hamiltonian parameters $(a, b)$ from the superconducting order parameters $[a, b]$.

\section{SUPERCONDUCTING FITNESS ANALYSIS}

Within the standard weak-coupling formalism, the superconducting fitness analysis allows us to discuss the relative stability of superconducting states based on properties of the normal electronic state [19,20]. In particular, it was shown that the larger the average over the Fermi surfaces (FSs) of $\operatorname{Tr}\left[\hat{F}_{A}(\mathbf{k})^{\dagger} \hat{F}_{A}(\mathbf{k})\right]$, the more robust the superconducting instability and the higher the critical temperature. Here, $\hat{F}_{A}(\mathbf{k})=\tilde{H}_{0}(\mathbf{k}) \tilde{\Delta}(\mathbf{k})+\tilde{\Delta}(\mathbf{k}) \tilde{H}_{0}^{*}(-\mathbf{k})$ is the superconducting fitness matrix, written in terms of the normalized normalstate Hamiltonian $\tilde{H}_{0}(\mathbf{k})=\left[\hat{H}_{0}(\mathbf{k})-h_{00}(\mathbf{k}) \tau_{0} \otimes \sigma_{0}\right] /|\mathbf{h}(\mathbf{k})|$, where $|\mathbf{h}(\mathbf{k})|=\sqrt{\sum_{(a, b) \neq(0,0)}\left|h_{a b}(\mathbf{k})\right|^{2}}$, and the normalized gap matrix $\tilde{\Delta}(\mathbf{k})$ with $\langle\tilde{\Delta}(\mathbf{k})\rangle_{\mathrm{FS}}=1$.

The superconducting fitness analysis for $\mathrm{CeRh}_{2} \mathrm{As}_{2}$ is summarized in Table IV. We start discussing the scenario with dominant ILH, such that we assume $\left|t_{p}\right|>\left|t_{u}+t_{d}\right|>\mid t_{u}-$ $t_{d}|>| \alpha|>| \lambda \mid$. From Table IV, we find that Cooper pairs with $a=0$ are the most stable since they have the contribution from the two largest terms in the normal-state Hamiltonian, $(1,0)$ and $(2,0)$. These are followed by pairs with $a=1$, supported only by $(1,0)$ hopping, controlled by $\left(t_{u}+t_{d}\right)^{2}$. Pairs with $a=2$ are less stable since these are supported only by $(2,0)$, controlled by $\left(t_{u}-t_{d}\right)^{2}$, while pairs with $a=3$
TABLE IV. Superconducting fitness analysis for all order parameters. Each line corresponds to an order parameter labeled by $[a, b]$, as in Eq. (3). The numerical entries correspond to $\operatorname{Tr}\left[\hat{F}_{A}(\mathbf{k}, s)^{\dagger} \hat{F}_{A}(\mathbf{k}, s)\right]=4 \sum_{c d}\left(\right.$ table entry) $\left|h_{c d}(\mathbf{k}, s)\right|^{2} /|h(\mathbf{k}, s)|^{2}$, for each term $(c, d)$ in the normal-state Hamiltonian. The last two columns summarize the effects of ILH and SOC dropping the accompanying momentum dependence of the respective functions.

\begin{tabular}{lccccccc}
\hline \hline & $(1,0)$ & $(2,0)$ & $(3,1)$ & $(3,2)$ & $(3,3)$ & ILH & SOC \\
\hline$[0,0]$ & 1 & 1 & 1 & 1 & 1 & & $2 \alpha^{2}+\lambda^{2}$ \\
{$[0,1]$} & 1 & 1 & 1 & 0 & 0 & $2\left(t_{u}^{2}+t_{d}^{2}\right)$ & $\alpha^{2}$ \\
{$[0,2]$} & 1 & 1 & 0 & 1 & 0 & & $\alpha^{2}$ \\
{$[0,3]$} & 1 & 1 & 0 & 0 & 1 & & $\lambda^{2}$ \\
{$[1,0]$} & 1 & 0 & 0 & 0 & 0 & & 0 \\
{$[1,1]$} & 1 & 0 & 0 & 1 & 1 & $\left(t_{u}+t_{d}\right)^{2}$ & $\alpha^{2}+\lambda^{2}$ \\
{$[1,2]$} & 1 & 0 & 1 & 0 & 1 & & $\alpha^{2}+\lambda^{2}$ \\
{$[1,3]$} & 1 & 0 & 1 & 1 & 0 & & $2 \alpha^{2}$ \\
{$[2,0]$} & 0 & 1 & 0 & 0 & 0 & & 0 \\
{$[2,1]$} & 0 & 1 & 0 & 1 & 1 & $\left(t_{u}-t_{d}\right)^{2}$ & $\alpha^{2}+\lambda^{2}$ \\
{$[2,2]$} & 0 & 1 & 1 & 0 & 1 & & $\alpha^{2}+\lambda^{2}$ \\
{$[2,3]$} & 0 & 1 & 1 & 1 & 0 & & $2 \alpha^{2}$ \\
{$[3,0]$} & 0 & 0 & 1 & 1 & 1 & & $2 \alpha^{2}+\lambda^{2}$ \\
{$[3,1]$} & 0 & 0 & 1 & 0 & 0 & 0 & $\alpha^{2}$ \\
{$[3,2]$} & 0 & 0 & 0 & 1 & 0 & & $\alpha^{2}$ \\
{$[3,3]$} & 0 & 0 & 0 & 0 & 1 & & $\lambda^{2}$ \\
\hline \hline
\end{tabular}

are not stabilized by any hopping term in the normal-state Hamiltonian. Among the intra-SL pairs $(a=0,3)$, the most robust order parameters are the spin singlets, stabilized by the larger contribution stemming from both Rashba and Ising SOC. Among the inter-SL pairs $(a=1,2)$, the most stable order parameters are the spin triplet states with a $d$-vector along the $z$-direction, stabilized by Rashba SOC. Overall this analysis supports $[0,0]$ and $[1,3]$ as the two most robust superconducting states for the ILH-dominated scenario. Moving now to the SOC dominated scenario, we assume $\left|t_{p}\right|>|\alpha|>$ $\left|t_{u}+t_{d}\right|>\left|t_{u}-t_{d}\right|>|\lambda|$. For the intra-SL Cooper pairs, we find that SOC stabilizes spin singlet states $(b=0)$, while for inter-SL pairs SOC stabilizes triplet states with the $d$-vector along the $z$-axis $(b=3)$. Among the intra-SL pairs, a finite ILH stabilizes $[0,0]$, and among the inter-SL pairs it supports $[1,3]$. Note that these are the same order parameter candidates found for the ILH dominated scenario.

Within the rough assumption that pairing in all channels have the same strength, we can conclude that the trivial order parameter with matrix structure $[0,0]$, an even parity intra-SL spin singlet (BCS) state, is the absolutely most stable superconducting state since all terms in the normal-state Hamiltonian support the instability in this channel. We note here that within the assumption of $t_{u}=t_{d}$ and $\lambda=0$, the [1,3] order parameter candidate, an odd parity SL-symmetric spin triplet (SLS-ST), would have the same transition temperature. These are good candidates for the low-field superconducting phase of $\mathrm{CeRh}_{2} \mathrm{As}_{2}$.

In the presence of a magnetic field along the $z$-axis, the proposed PDW state, an odd parity intra-SL spin singlet, here captured by $[3,0]$, is not very robust since the normal state ILH terms $(1,0)$ and $(2,0)$ do not contribute to its stabilization. 
Interestingly, from the analysis above, within the assumption of $t_{u}=t_{d}$ and $\lambda=0$, the order parameter [2,3], an odd parity SL-antisymmetric spin triplet (SLA-ST), is equally stable. In fact, under the assumption $\left|t_{u}-t_{d}\right|>\lambda$, the SLA-ST is more robust than the PDW state. Order parameters with a similar nature, antisymmetric in an internal DOF, were recently proposed in multiple contexts [21-24].

\section{PHASE DIAGRAM}

With these facts at hand, we now examine the behavior under $z$-axis magnetic field of the four order parameters identified above. The hierarchy of energy scales motivates the writing of a quasiclassical theory assuming $\left|t_{p}\right| \gg\left\{\mid t_{u}+\right.$ $\left.t_{d}|,| t_{u}-t_{d} \mid, \alpha, \lambda\right\}$. For simplicity, here we take $t_{u}=t_{d}$ and $\lambda=0$ and study the interplay of the magnetic field $B$, Rashba SOC $\alpha$, and ILH $\left|t_{u}+t_{d}\right|$. We extend the linearized Eilenberger equations of Ref. [25] to include $\left|t_{u}+t_{d}\right|$, which allows us to obtain the transition lines of the best low and high field phase candidates. For each pairing irrep-channel, we associate a superconducting critical temperature $T_{\text {irrep }}$ that is defined in the absence of magnetic field and SOC. For simplicity, we consider the same critical temperatures for all fitness-favored channels by setting $T_{A_{1 g}}=T_{A_{2 g}}=T_{A_{1 u}}=T_{A_{2 u}}=T_{c}$. We find two promising scenarios that display the same phase diagram: (i) intra-SL scenario, with a transition from an even BCS state [0,0] to a high field odd PDW state [3,0]; (ii) inter-SL scenario, with a transition from a low field odd SLS-ST state $[1,3]$ to a high field odd SLA-ST state $[2,3]$. These results are summarized in Fig. 2. In both scenarios, a first-order phase transition separates the high from the low field phase. The exact location of these transitions requires a treatment beyond linearization.

\section{DISCUSSION}

Given the extremely large upper critical field observed experimentally, the results above suggest that the normal state is SOC dominated. Note, though, that this limit does not allow us to distinguish between the intra-SL and inter-SL scenarios, as illustrated by Fig. 2. One potential way to distinguish them is the effect of impurities. Current samples of $\mathrm{CeRh}_{2} \mathrm{As}_{2}$ are likely to be in the dirty limit [1], in which case a k-independent order parameter in the microscopic basis would be robust [25-28]. This suggests the intra-SL scenario for the low field phase since, in the inter-SL, $d_{13}(\mathbf{k})$ is odd in momentum. If cleaner samples become available and display a significantly enhanced $T_{c}$, then the inter-SL scenario would remain a good candidate. Furthermore, the presence and location of nodes in the superconducting gap can give us important information since the inter-SL scenario involves odd-parity states which necessarily display line nodes in the superconducting gap at $k_{z}=0$.

In this work, we do not discuss the pairing mechanism. For a realistic discussion, details of the FS in the heavy fermion regime are needed to investigate possible spin-fluctuation mechanisms. Also, the clarification of the nature of the hidden order observed at $0.4 \mathrm{~K}$ and its association with multipolar order brings an interesting possibility for exotic pairing from multipolar interactions [29-31]. Moreover, the presence of

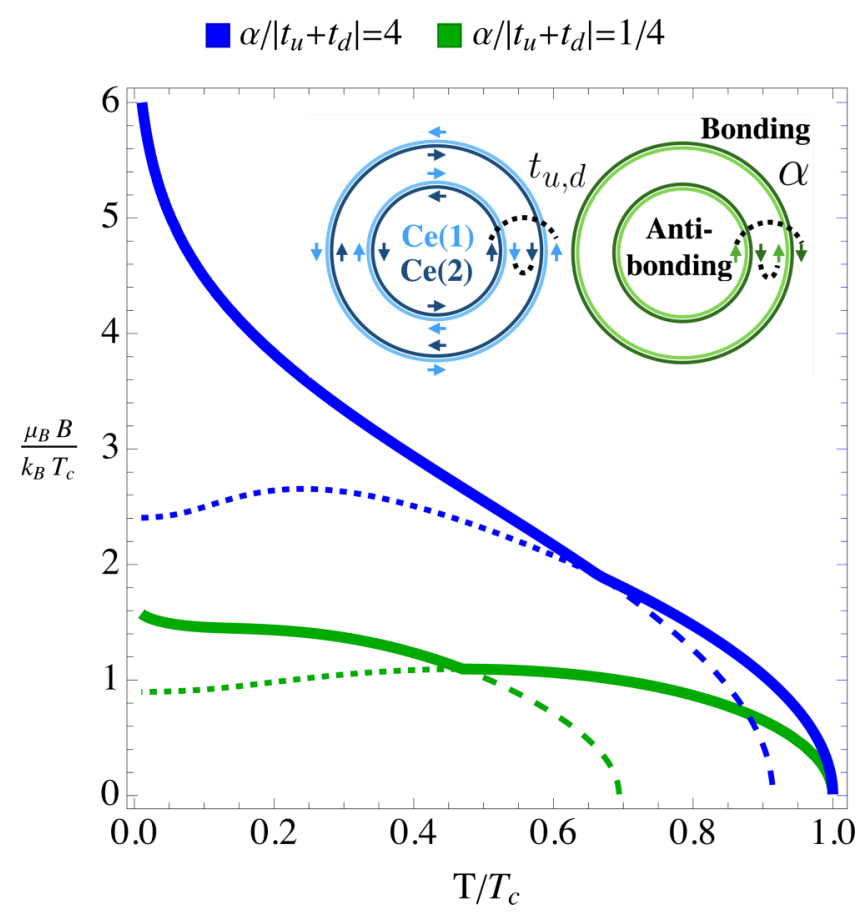

FIG. 2. Identical temperature versus $z$-axis magnetic field phase diagrams for the even-odd intra-SL $[0,0] \rightarrow[3,0]$ and odd-odd inter-SL $[1,3] \rightarrow[2,3]$ scenarios with $\left|t_{u}+t_{d}\right|=T_{c}$. The blue curves correspond to the SOC-dominated regime, while the green curves correspond to the ILH-dominated regime. The dotted (dashed) lines are the extensions of the low (high) field solutions. The inset displays schematic two-dimensional cuts of the FSs. Left: SOCdominated regime. The lighter (darker) color indicates FSs stemming from $\mathrm{Ce}(1)[\mathrm{Ce}(2)]$ layers, and the arrows indicate the helicity. Turning on ILH connects FSs with same helicity and complementary Ce-content (dotted lines). Right: ILH-dominated regime. The lighter (darker) color indicates spin up (down). Turning on SOC connects FSs with opposite spin and bonding state (dotted lines).

quantum critical behavior evidenced by the temperature dependence of the specific heat at low temperatures suggests a scenario similar to $\beta-\mathrm{YbAlB}_{4}$, for which a careful description of the crystal electric field states was key to understand its phenomenology [32-35].

Future studies targeting the vortex core size through the first-order transition and possible zero-energy states can further elucidate and distinguish the two scenarios. From thermodynamics, one expects a sudden change of the core size through the transition $[14,36]$, which would give further evidence of two distinct phases. For the triplet scenario, one expects zero-energy bound states for both low and high field phases. In contrast, for the singlet scenario, the zero-energy states only exist in the high field phase [36-38]. Therefore, the presence/absence of zero-energy states in the low field phase could distinguish between the triplet/singlet scenarios.

\section{CONCLUSION}

In summary, we analyzed all possible superconducting order parameters for $\mathrm{CeRh}_{2} \mathrm{As}_{2}$ within the $\mathrm{Ce}$-sites perspective. We find temperature versus $z$-axis magnetic field phase diagrams in qualitative agreement with experiments for both 
SOC- and ILH-dominated normal states. We identified two possible scenarios for the two superconducting phases observed in $\mathrm{CeRh}_{2} \mathrm{As}_{2}$ : (i) even-odd intra-SL, associated with the previously proposed PDW scenario and (ii) new odd-odd inter-SL scenario. Our work stimulates more efforts in the field of complex locally noncentrosymmetric materials with extra internal degrees of freedom. We have preliminary evidence that the joint action of SOC and magnetic field might lead singlet-triplet mixing in both the low and high field phases. We detail this possibility in a future, more extended paper. Further theoretical work analyzing the interplay of normal-state parameters and magnetic field, as well as topological aspects are interesting directions for future work.
Note added. Recently, we became aware of the preprint [39] which suggests that in-plane magnetic fields would be able to discriminate the two scenarios proposed here, favoring the BCS-PDW scenario.

\section{ACKNOWLEDGMENTS}

The authors acknowledge M. Fischer, E. Hassinger, and Y.-P. Huang for useful discussions. A.R. acknowledges support from the SNSF Ambizione grant. A.R. and D.M. thank the support of the Condensed Matter Theory Group at the Paul Scherrer Institute that allowed this collaboration to be established.
[1] S. Khim, J. F. Landaeta, J. Banda, N. Bannor, M. Brando, P. M. R. Brydon, D. Hafner, R. Küchler, R. Cardoso-Gil, U. Stockert, A. P. Mackenzie, D. F. Agterberg, C. Geibel, and E. Hassinger, Field-induced transition from even to odd parity superconductivity in $\mathrm{CeRh}_{2} \mathrm{As}_{2}$, arXiv:2101.09522.

[2] R. A. Fisher, S. Kim, B. F. Woodfield, N. E. Phillips, L. Taillefer, K. Hasselbach, J. Flouquet, A. L. Giorgi, and J. L. Smith, Specific Heat of $\mathrm{Upt}_{3}$ : Evidence for Unconventional Superconductivity, Phys. Rev. Lett. 62, 1411 (1989).

[3] S. Adenwalla, S. W. Lin, Q. Z. Ran, Z. Zhao, J. B. Ketterson, J. A. Sauls, L. Taillefer, D. G. Hinks, M. Levy, and B. K. Sarma, Phase Diagram of $\mathrm{UPt}_{3}$ from Ultrasonic Velocity Measurements, Phys. Rev. Lett. 65, 2298 (1990).

[4] R. Joynt and L. Taillefer, The superconducting phases of $\mathrm{UPt}_{3}$, Rev. Mod. Phys. 74, 235 (2002).

[5] S. Ran, I. L. Liu, Y. S. Eo, D. J. Campbell, P. M. Neves, W. T. Fuhrman, S. R. Saha, C. Eckberg, H. Kim, D. Graf, F. Balakirev, J. Singleton, J. Paglione, and N. P. Butch, Extreme magnetic field-boosted superconductivity, Nat. Phys. 15, 1250 (2019).

[6] J. S. Van Dyke, F. Massee, M. P. Allan, J. C. S. Davis, C. Petrovic, and D. K. Morr, Direct evidence for a magnetic f-electron-mediated pairing mechanism of heavy-fermion superconductivity in cecoin5, Proc. Natl. Acad. Sci. USA 111, 11663 (2014).

[7] J. F. Landaeta, D. Subero, D. Catalá, S. V. Taylor, N. Kimura, R. Settai, Y. Ōnuki, M. Sigrist, and I. Bonalde, Unconventional superconductivity and quantum criticality in the heavy fermions ceirsi $i_{3}$ and cerhsi ${ }_{3}$, Phys. Rev. B 97, 104513 (2018).

[8] B. White, J. Thompson, and M. Maple, Unconventional superconductivity in heavy-fermion compounds, Physica C 514, 246 (2015).

[9] M. Smidman, O. Stockert, J. Arndt, G. M. Pang, L. Jiao, H. Q. Yuan, H. A. Vieyra, S. Kitagawa, K. Ishida, K. Fujiwara, T. C. Kobayashi, E. Schuberth, M. Tippmann, L. Steinke, S. Lausberg, A. Steppke, M. Brando, H. Pfau, U. Stockert, P. Sun et al., Interplay between unconventional superconductivity and heavy-fermion quantum criticality: $\mathrm{CeCu}_{2} \mathrm{Si}_{2}$ versus $\mathrm{YbRh}_{2} \mathrm{Si}_{2}$, Philos. Mag. 98, 2930 (2018).

[10] M. H. Fischer, F. Loder, and M. Sigrist, Superconductivity and local noncentrosymmetricity in crystal lattices, Phys. Rev. B 84, 184533 (2011).

[11] D. Maruyama, M. Sigrist, and Y. Yanase, Locally noncentrosymmetric superconductivity in multilayer systems, J. Phys. Soc. Jpn. 81, 034702 (2012).
[12] T. Yoshida, M. Sigrist, and Y. Yanase, Parity-mixed superconductivity in locally non-centrosymmetric system, J. Phys. Soc. Jpn. 83, 013703 (2014).

[13] D. Möckli, Y. Yanase, and M. Sigrist, Orbitally limited pairdensity-wave phase of multilayer superconductors, Phys. Rev. B 97, 144508 (2018).

[14] E. G. Schertenleib, M. H. Fischer, and M. Sigrist, Unusual $H-T$ phase diagram of $\mathrm{CeRh}_{2} \mathrm{As}_{2}$ : The role of staggered non-centrosymmetricity, Phys. Rev. Research 3, 023179 (2021).

[15] A. Ptok, K. J. Kapcia, P. T. Jochym, J. Łażewski, A. M. Oleś, and P. Piekarz, Electronic and dynamical properties of $\mathrm{CeRh}_{2} \mathrm{As}_{2}$ : Role of $\mathrm{Rh}_{2} \mathrm{As}_{2}$ layers and expected hidden orbital order, arXiv:2102.02735.

[16] J. Arndt, O. Stockert, K. Schmalzl, E. Faulhaber, H. S. Jeevan, C. Geibel, W. Schmidt, M. Loewenhaupt, and F. Steglich, Spin Fluctuations in Normal State $\mathrm{CeCu}_{2} \mathrm{Si}_{2}$ on Approaching the Quantum Critical Point, Phys. Rev. Lett. 106, 246401 (2011).

[17] F. Steglich, J. Arndt, O. Stockert, S. Friedemann, M. Brando, C. Klingner, C. Krellner, C. Geibel, S. Wirth, S. Kirchner, and Q. $\mathrm{Si}$, Magnetism, f-electron localization and superconductivity in 122-type heavy-fermion metals, J. Phys.: Condens. Matter 24, 294201 (2012).

[18] V. Cvetkovic and O. Vafek, Space group symmetry, spin-orbit coupling, and the low-energy effective hamiltonian for ironbased superconductors, Phys. Rev. B 88, 134510 (2013).

[19] A. Ramires and M. Sigrist, Identifying detrimental effects for multiorbital superconductivity: Application to $\mathrm{Sr}_{2} \mathrm{RuO}_{4}$, Phys. Rev. B 94, 104501 (2016).

[20] A. Ramires, D. F. Agterberg, and M. Sigrist, Tailoring $T_{c}$ by symmetry principles: The concept of superconducting fitness, Phys. Rev. B 98, 024501 (2018).

[21] H. G. Suh, H. Menke, P. M. R. Brydon, C. Timm, A. Ramires, and D. F. Agterberg, Stabilizing even-parity chiral superconductivity in $\mathrm{Sr}_{2} \mathrm{RuO}_{4}$, Phys. Rev. Research 2, 032023(R) (2020).

[22] S. K. Ghosh, G. Csire, P. Whittlesea, J. F. Annett, M. Gradhand, B. Újfalussy, and J. Quintanilla, Quantitative theory of triplet pairing in the unconventional superconductor $\mathrm{LaNiGa}_{2}$, Phys. Rev. B 101, 100506(R) (2020).

[23] O. Vafek and A. V. Chubukov, Hund Interaction, Spin-Orbit Coupling, and the Mechanism of Superconductivity in Strongly Hole-Doped Iron Pnictides, Phys. Rev. Lett. 118, 087003 (2017). 
[24] L. Fu and E. Berg, Odd-Parity Topological Superconductors: Theory and Application to $\mathrm{Cu}_{x} \mathrm{Bi}_{2} \mathrm{Se}_{3}$, Phys. Rev. Lett. 105, 097001 (2010).

[25] D. Möckli and M. Khodas, Ising superconductors: Interplay of magnetic field, triplet channels, and disorder, Phys. Rev. B 101, 014510 (2020).

[26] D. Möckli and M. Khodas, Robust parity-mixed superconductivity in disordered monolayer transition metal dichalcogenides, Phys. Rev. B 98, 144518 (2018).

[27] D. Möckli, M. Haim, and M. Khodas, Magnetic impurities in thin films and 2d ising superconductors, J. Appl. Phys. 128, 053903 (2020).

[28] L. Andersen, A. Ramires, Z. Wang, T. Lorenz, and Y. Ando, Generalized anderson's theorem for superconductors derived from topological insulators, Sci. Adv. 6, eaay6502 (2020).

[29] A. Sakai, K. Kuga, and S. Nakatsuji, Superconductivity in the ferroquadrupolar state in the quadrupolar kondo lattice $\operatorname{PrTi}_{2} \mathrm{Al}_{20}$, J. Phys. Soc. Jpn. 81, 083702 (2012).

[30] M. Tsujimoto, Y. Matsumoto, T. Tomita, A. Sakai, and S. Nakatsuji, Heavy-Fermion Superconductivity in the Quadrupole Ordered State of $\operatorname{PrV}_{2} \mathrm{Al}_{20}$, Phys. Rev. Lett. 113, 267001 (2014).

[31] H. Kotegawa, M. Yogi, Y. Imamura, Y. Kawasaki, G.-Q. Zheng, Y. Kitaoka, S. Ohsaki, H. Sugawara, Y. Aoki, and H. Sato, Evidence for Unconventional Strong-Coupling Superconductivity in $\mathrm{PrOs}_{4} \mathrm{Sb}_{12}$ : An Sb Nuclear Quadrupole Resonance Study, Phys. Rev. Lett. 90, 027001 (2003).
[32] S. Nakatsuji, K. Kuga, Y. Machida, T. Tayama, T. Sakakibara, Y. Karaki, H. Ishimoto, S. Yonezawa, Y. Maeno, E. Pearson, G. G. Lonzarich, L. Balicas, H. Lee, and Z. Fisk, Superconductivity and quantum criticality in the heavy-fermion system $\beta$-YbAlB 4 , Nat. Phys. 4, 603 (2008).

[33] Y. Matsumoto, S. Nakatsuji, K. Kuga, Y. Karaki, N. Horie, Y. Shimura, T. Sakakibara, A. H. Nevidomskyy, and P. Coleman, Quantum criticality without tuning in the mixed valence compound $\beta$-YbAlB4, Science 331, 316 (2011).

[34] A. Ramires, P. Coleman, A. H. Nevidomskyy, and A. M. Tsvelik, $\beta-\mathrm{YbAlB}_{4}$ : A Critical Nodal Metal, Phys. Rev. Lett. 109, 176404 (2012).

[35] A. Ramires and P. Coleman, Theory of the Electron Spin Resonance in the Heavy Fermion Metal $\beta-\mathrm{YbAlB}_{4}$, Phys. Rev. Lett. 112, 116405 (2014).

[36] Y. Higashi, Y. Nagai, T. Yoshida, Y. Masaki, and Y. Yanase, Robust zero-energy bound states around a pair-density-wave vortex core in locally noncentrosymmetric superconductors, Phys. Rev. B 93, 104529 (2016).

[37] T. Yoshida, M. Sigrist, and Y. Yanase, Topological Crystalline Superconductivity in Locally Noncentrosymmetric Multilayer Superconductors, Phys. Rev. Lett. 115, 027001 (2015).

[38] K. Nogaki, A. Daido, J. Ishizuka, and Y. Yanase, Topological crystalline superconductivity in locally noncentrosymmetric $\mathrm{CeRh}_{2} \mathrm{As}_{2}$, arXiv:2103.08088.

[39] A. Skurativska, M. Sigrist, and M. H. Fischer, Spin response and topology of a staggered Rashba superconductor, arXiv:2103.06282 